\title{
Targeted drug delivery into reversibly injured myocardium with silica nanoparticles: surface functionalization, natural biodistribution, and acute toxicity
}

\author{
This article was published in the following Dove Press journal: \\ International Journal of Nanomedicine \\ 31 March 2010 \\ Number of times this article has been viewed
}

\author{
Michael M Galagudza' \\ Dmitry V Korolev' \\ Dmitry L Sonin' \\ Viktor N Postnov ${ }^{2}$ \\ Garry V Papayan ${ }^{3}$ \\ Ivan S Uskov' \\ Anastasia $\bigvee$ Belozertseva \\ Eugene V Shlyakhto' \\ 'Institute of Experimental Medicine, \\ VA. Almazov Federal Heart, \\ Blood and Endocrinology Center, \\ St-Petersburg, Russian Federation; \\ ${ }^{2}$ Chemical Faculty, St-Petersburg \\ State University, St-Petersburg, \\ Russian Federation; ${ }^{3}$ Department \\ of Pathophysiology, IP. Pavlov State \\ Medical University, St-Petersburg, \\ Russian Federation
}

\begin{abstract}
The clinical outcome of patients with ischemic heart disease can be significantly improved with the implementation of targeted drug delivery into the ischemic myocardium. In this paper, we present our original findings relevant to the problem of therapeutic heart targeting with use of nanoparticles. Experimental approaches included fabrication of carbon and silica nanoparticles, their characterization and surface modification. The acute hemodynamic effects of nanoparticle formulation as well as nanoparticle biodistribution were studied in male Wistar rats. Carbon and silica nanoparticles are nontoxic materials that can be used as carriers for heart-targeted drug delivery. Concepts of passive and active targeting can be applied to the development of targeted drug delivery to the ischemic myocardial cells. Provided that ischemic heart-targeted drug delivery can be proved to be safe and efficient, the results of this research may contribute to the development of new technologies in the pharmaceutical industry.
\end{abstract}

Keywords: nanocarriers, targeted drug delivery, myocardial ischemia

\section{Introduction}

The concept of targeted drug delivery (TDD) implies selective accumulation of a drug within the affected tissue after systemic administration of the carrier-bound drug with the minimal possible side effects on irrelevant organs and tissues. ${ }^{1}$ The idea of TDD was first introduced by Paul Erlich in 1906, who put forward the concept of the "magic bullet" directed against the target cell alone, with no damage to healthy cells. ${ }^{2}$ Site-targeted nanosized particles as drug carriers may offer an advantage of safety and efficacy superior to that of more routine prototypes.

A typical nanoparticulate carrier for TDD includes several functional elements. Its major part is the drug-loaded nanoparticle. An important step in its fabrication is the functionalization of the particle, which implies the binding of the targeting ligand to the particle surface. The ligand should ensure specific interaction of the particle with the complementary molecule, whenever delivered to the target cell. The nanoparticles can also be bound to radioactive isotopes or fluorescent dyes, which allow their visualization in the tissue of interest. Effective TDD with use of nanoparticulate carriers can bring solutions to such problems as drug toxicity, insufficient bioavailability, poor solubility and stability.

To date, the research on TDD has been mostly concentrated on the development of tumor-targeted nanomedicines. It might be hypothesized that TDD strategy approved
Correspondence: Michael Galagudza Institute of Experimental Medicine, V.A. Almazov Federal Heart, Blood and Endocrinology Center, Akkuratova str. 2, St-Petersburg, Russian Federation

$\mathrm{Tel}+78124997035$

Fax +78124997069

Email galagoudza@mail.ru 
in oncology can be applied in other clinical fields, not for destruction of specific tissue, more for the salvage of reversibly injured cells; - in particular cells destined to die within the area of myocardial ischemia-reperfusion. One reasonable approach to the problem may be targeted intramyocardial delivery of the agents (such as certain angiogenic growth factors, erythropoietin, adenosive triphosphate (ATP) -sensitive potassium channel openers), either covalently and/or noncovalently bound to the nanoparticulate carriers. One may suppose that local accumulation of drug-loaded nanoparticles within the ischemic area of the heart can at least in principle be achieved by selective binding of annexin $\mathrm{V}$ to the particle surface. Annexin V is a naturally expressed soluble protein that binds to phosphatidylserine head in a calcium-dependent fashion. Phosphatidylserine is normally localized to the inner surface of the plasma membrane, but when sublethal cell injury occurs it is translocated to the external layer the cell surface. ${ }^{3}$ Annexin V is widely used as a marker protein for the visualization of cell injury and apoptotic death in vitro. Specific interaction of the nanoparticle-anchored annexin $\mathrm{V}$ with phosphatidylserine would potentially ensue the binding of nanoparticles to the cardiomyocyte membrane with their subsequent accumulation in the area of interest.

Apart from the active heart targeting by annexin V, we cannot rule out the probability that passive targeting may be helpful in treating ischemic-reperfused myocardium. ${ }^{4}$ The basic idea is that the nanoparticulate carriers can be retained and accumulated within the ischemic-reperfused area of the heart owing to increased microvascular permeability to all macromolecules, the nanocarrier including. ${ }^{5}$ In this regard, the functional characteristics of myocardial microvessels are more or less similar to those of the tumor, which would make the proposed approach even closer to that already practiced.

In this study, we provide preliminary evidence that $10 \mathrm{~nm}$ silica nanoparticles (SNP) might be used for heart-targeted drug delivery in both passive and active ways as described above. The study was aimed at the development of a technique of SNP functionalization, evaluation of SNP effects on the parameters of systemic hemodynamics, and investigation of SNP biodistribution using optical fluorescence.

\section{Materials and methods}

\section{Preparation and characterization of SNP}

The standard pyrogenic highly dispersed silica (Aerosil ${ }^{\circledR}$ of A170, A300, and A380 marks obtained from Vekton ${ }^{\mathrm{TM}}$, St Petersburg, Russia) was used throughout the experiments. The surface area of SNP was determined using the
Brunauer-Emmett-Teller (BET) method and averaged from 170 to $380 \mathrm{~m}^{2} / \mathrm{g}$. The mean particle diameter varied from 6 to $13 \mathrm{~nm}$. The technique of SNP functionalization included three sequential steps: modification of SNP with (3-aminopropyl)triethoxysilane, hydrolysis of unreacted alkoxysilane groups, and binding of fluorescein. Chemosorption of (3-aminopropyl)triethoxysilane was performed from the gaseous phase with use of dried nitrogen as a carrier gas. The synthesis was done in the vertical quartz flow reactor at $220^{\circ} \mathrm{C}$ for 2 hours. Hydrolysis of the unreacted alkoxysilane groups was achieved with water vapor at $150^{\circ} \mathrm{C}$ for 1 hour. The covalent binding of fluorescein to the aminated silica was done using carbodiimide technique for 1 hour (Scheme 1 and 2). All chemicals were purchased from Sigma-Aldrich Chemical Co (Moscow, Russia). The surface reactions were controlled with infrared spectroscopy (diffuse reflection) (Perkin-Elmer 1760×). The amount of fluorescein bound to the surface of SNP was determined spectrophotometrically at $\lambda=490 \mathrm{~nm}$. Fluorescein content within the samples of modified SNP varied from 0.01 to $0.02 \mathrm{mM} / \mathrm{g}$. Another fluorescent dye that can be used for labeling of SNP is considered to be indocyanine green (ICG). This fluorophore has an absorption band in the near-infrared region $(\lambda=780 \mathrm{~nm})$. We have developed the technique of indocyanine green immobilization on the surface of aminated silica. First of all, it has been established that the sample obtained is stable in the saline to be used for the evaluation of nanoparticle biodistribution. Then, fluorescently labeled SNP were used for biodistribution studies (see below).

\section{Acute toxicity and biodistribution}

We studied in the rat model the acute effect of nanoparticle formulations on basic hemodynamic parameters. For this purpose, the suspensions of silica $\left(\mathrm{nSiO}_{2}\right)$, fluorescein-doped silica ( $\mathrm{nSiO}_{2}+$ fluorescein), and carbon $(\mathrm{nC})$ nanoparticles in $0.9 \%$ sodium hydrochloride solution were prepared. In all suspensions, the final concentration of either silica or carbon was $2 \mathrm{mg} / \mathrm{mL}$. The binding of fluorescein to the nanoparticle surface was performed using the chemical assembly method described above. Fluorescein concentration in the suspension of $\mathrm{nSiO}_{2}+$ fluorescein was about $0.013 \mathrm{mg} / \mathrm{mL}$.

Male Wistar rats, 250 to $300 \mathrm{~g}$ body weight were used throughout the experiments. The animals were fed regular chow, and water was available ad libitum. All experiments were performed in accordance with the Guide for the Care and Use of Laboratory Animals (publication No. [NIH] 85-23). The animals were anesthetized with sodium pentobarbital $(60 \mathrm{mg} / \mathrm{kg})$. The trachea was intubated through 


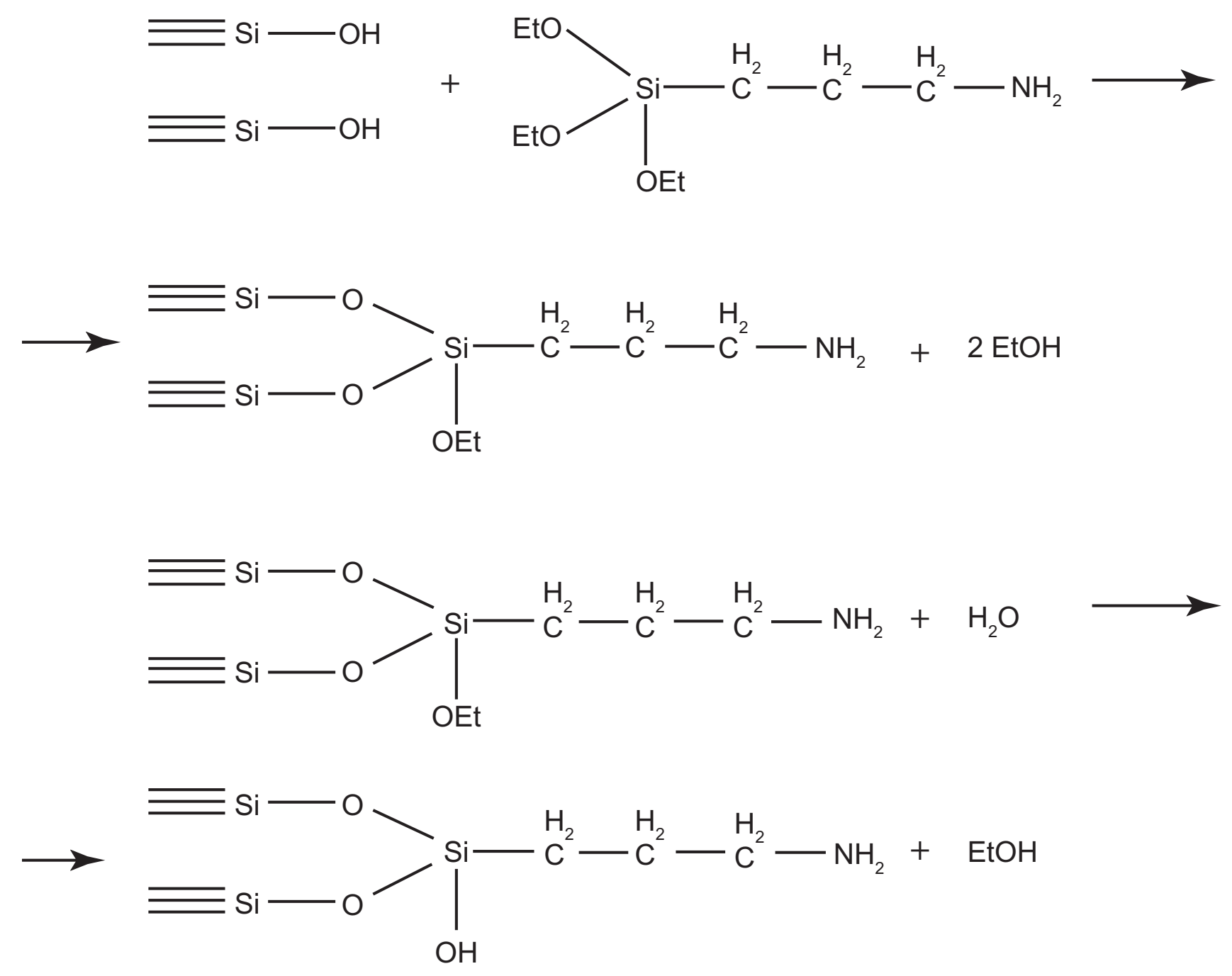

Scheme I Aerosil surface functionalization using chemical assembly method.

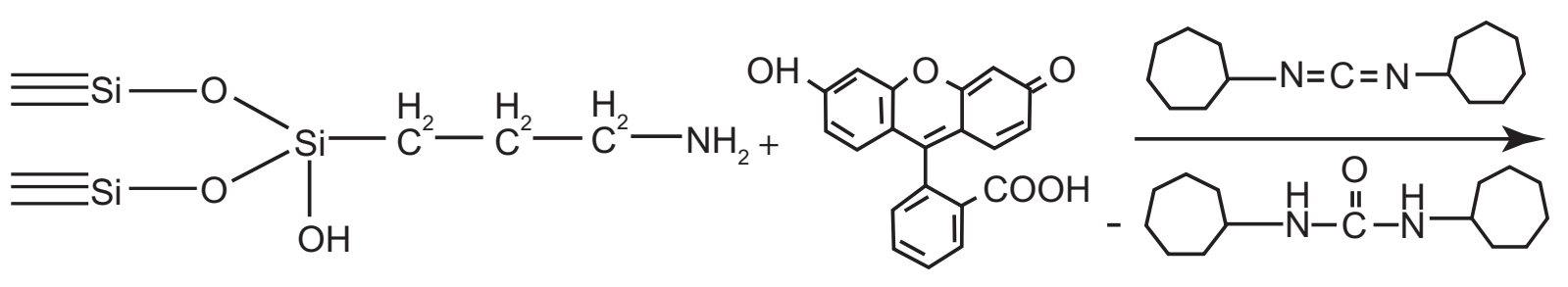<smiles>C#CO[Si](O)(CCCCNC(=O)c1ccccc1-c1c2ccc(=O)cc-2oc2cc(O)ccc12)O[Si]#C</smiles>

Scheme 2 Covalent binding of fluorescein to the aminated surface of silica nanoparticle. 
a cervical incision. In spontaneously breathing animals the right carotid artery and femoral vein were cannulated for blood pressure monitoring and anesthesia maintenance, respectively. The other femoral vein was cannulated for infusion of the solutions to be tested. Measurements of heart rate (HR), mean arterial pressure (MAP) and pulse arterial pressure (PAP) were done in all groups at baseline, that is, 2 minutes before the first infusion. The volume of the first infusion for any tested nanoparticle formulation was $0.2 \mathrm{~mL}$, while for two following infusions it was $0.4 \mathrm{~mL}$, so that the total volume was $1 \mathrm{~mL}(0.7 \mathrm{mg}$ of nanoparticulate carrier per $100 \mathrm{~g}$ of the animal body weight). The intravenous infusions of the equivalent amounts of $0.9 \%$ sodium hydrochloride were performed in the controls. In all groups, the infusions were separated by a 20 minute rest period. The hemodynamic responses to infusions were evaluated at the next time points: 2 minutes after initiation of each infusion, by the end of each infusion, as well as 10 minutes and 20 minutes following each infusion (Figure 1).

The biodistribution of SNP was determined by fluorescence imaging. Fluorescein and indocyanine green were used for labeling of SNP. The animals ( $\mathrm{n}=3$ for each fluorescent dye) received intravenous infusion of fluorescently labeled nanoparticles suspended in normal saline at a volume of $1 \mathrm{~mL}$ totally for 10 minutes. Twenty minutes after the end of each infusion, the animals were sacrificed by an overdose of anesthesia, and heart, brain, liver, spleen, lung, and kidney were removed for subsequent measurements. Control animals $(n=3)$ were treated with the vehicle alone. The fluorescence was registered using the home-made FLUM3 setup equipped with mercury lamp illuminator and TV camera. The fluorescence of fluorescein- and indocyanine green-labeled nanoparticles was elicited using band-pass interference filters of 435 and $780 \mathrm{~nm}$, respectively. The images obtained in the experiments with fluorescein-labeled nanoparticles were split into red, green, blue (RGB) channels. Channel G has been chosen as a target one. The relative change in the fluorescence intensity (percentage change as related to the control, $\Delta \mathrm{G} \%$ ) has been calculated as follows: $\Delta \mathrm{G}=(\mathrm{G} 2-\mathrm{G} 1) / \mathrm{G} 1$, where G1 is the level of (auto)fluorescence in controls, G2 - the fluorescence intensity of samples obtained from the animals treated with $\mathrm{nSiO}_{2}+$ fluorescein. In the experiments with indocyanine green-labeled nanoparticles, black-and-white images were obtained, and the relative change in the fluorescence level (ie, percentage change from control, $\Delta \mathrm{F} \%$ ) was calculated as described above. Measurement of fluorescence intensity was performed with use of Ecoskin software 1.0.1. Statistical analysis was performed by the Mann-Whitney $U$ test, with $P$ values $<0.05$ considered significant.

\section{Results}

\section{Acute effects of nanoparticle suspensions on hemodynamic parameters}

Hemodynamic data are summarized in Table 1. Briefly, in all animal groups, there were no significant alterations in heart rate throughout the trials. The first infusion of any nanoparticle formulation tested had no effect on hemodynamic parameters (see also Figure 2). Furthermore, there was no significant difference in MAP between the baseline time point and the end of the experiment. However, both second and third infusions of $\mathrm{nSiO}_{2}$ evoked transient increase of MAP (Figure 2). Infusions of $\mathrm{nC}$ or saline alone did not affect the MAP values assayed. There was a significant increase in PAP of the animals that received $\mathrm{nSiO}_{2}$ or $\mathrm{nC}$ at the end of the observation. Administration of $\mathrm{nSiO}_{2}+$ fluorescein resulted in an increase of PAP too, while PAP in the controls remained unaffected throughout the experiment (Table 1).

Thus, intravenous infusion of distinct nanoparticle formulations caused mild changes in systemic hemodynamics which appears to be indicative of the appropriate biocompatibility of nanomaterials tested.

\section{Biodistribution}

The biodistribution data are shown in Table 2. It should be noted that exposure of the organ samples to UV light $(\lambda=435 \mathrm{~nm})$ for detection of fluorescein-doped nanoparticles was associated

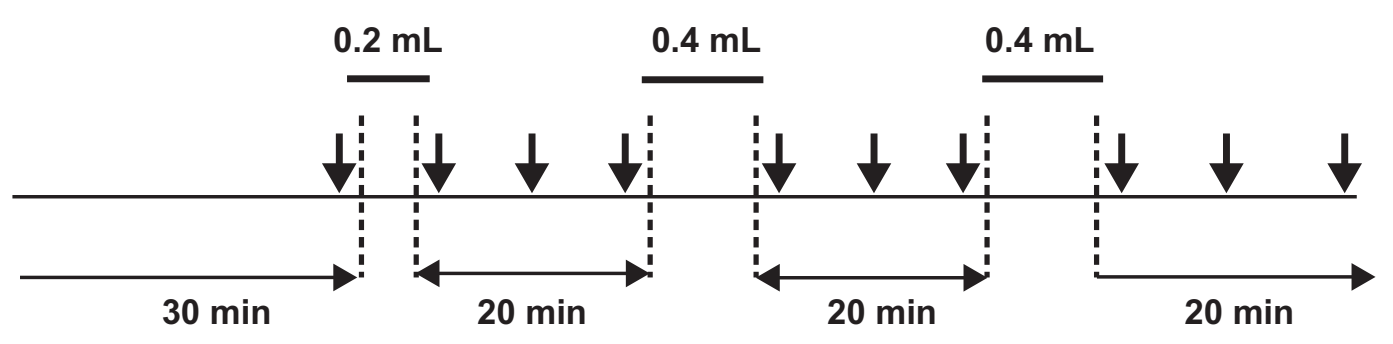

Figure I Experimental protocol. $\downarrow-$ time point of evaluation of hemodynamic parameters. 


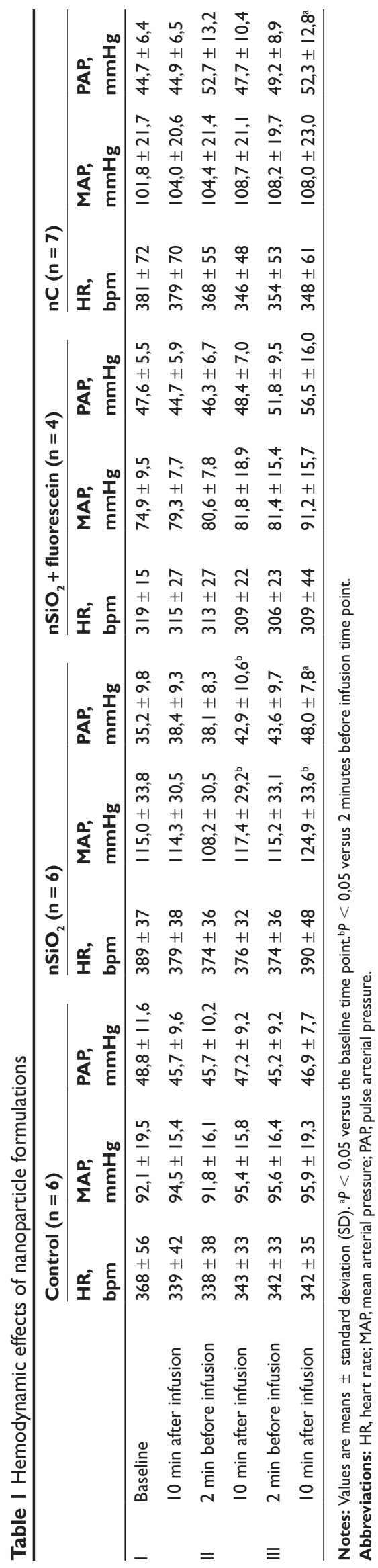

with significant autofluorescence that may interfere with the signal of interest. We nevertheless were able to discriminate statistically between meaningful and background signals to show the predominant accumulation of fluorescein-labeled nanoparticles within the lung, spleen and liver, that is, in the organs of RES. Besides, indocyanine green has proved to be helpful for the avoidance of tissue excessive autofluorescence at $\lambda=780 \mathrm{~nm}$. Concerning indocyanine green-labeled nanoparticles, the highest accumulation was within the liver and kidney compared to other organs. Therefore, for fluorescent labeling of SNP and investigation of their biodistribution the infrared fluorophores appear to be preferable.

\section{Discussion}

Although the idea of TDD to the ischemic heart put forward in this paper seems to be justified, at least in principle, the following problems lie ahead. Since systemic administration of drug nanocarriers results in their selective accumulation in the targets under conditions of normal blood flow, it remains to be solved in what way their effective concentration can be achieved in the ischemic hearts. Supposedly, the increased residence time of nanocarriers within the normally perfused tissue adjacent to ischemic one would be the solution if one takes into consideration the sufficient diffusion between the two. An alternative solution may be an increase in payloading of each nanoparticle with drugs and/or targeting ligands. It should be borne in mind, however, that such a strategy might be at substantial risk of the competitive uptake of nanocarriers by the immune system at the expense of the targets.

Insufficient evidence is available on the clinical effectiveness of nanoparticulate drug carriers. More studies are required to elucidate the pharmacokinetics of nanocarriers, their biodistribution and putative toxicity. All of the newly developed nanosystems for targeted delivery demonstrate unique properties and need careful testing. The refinement of the techniques of nanocarrier fabrication is under way and aimed primarily at ensuring better uptake of nanoparticles by the target cells, formation of a higher gradient of drug concentration between injured and unaffected tissue, and lowering drug toxicity. The solution of these problems would be a major step in the development of the "ideal tool" for well-addressed delivery of the drug into the tissue of interest.

In the current study, it was shown that intravenous infusion of SNP is associated with mild changes in hemodynamic parameters. This is indicative of the lack of acute toxicity of the nanoparticle formulations tested. An important issue related to the potential use of SNP for medical applications is their biocompatibility and biodegradability. It has been found before 


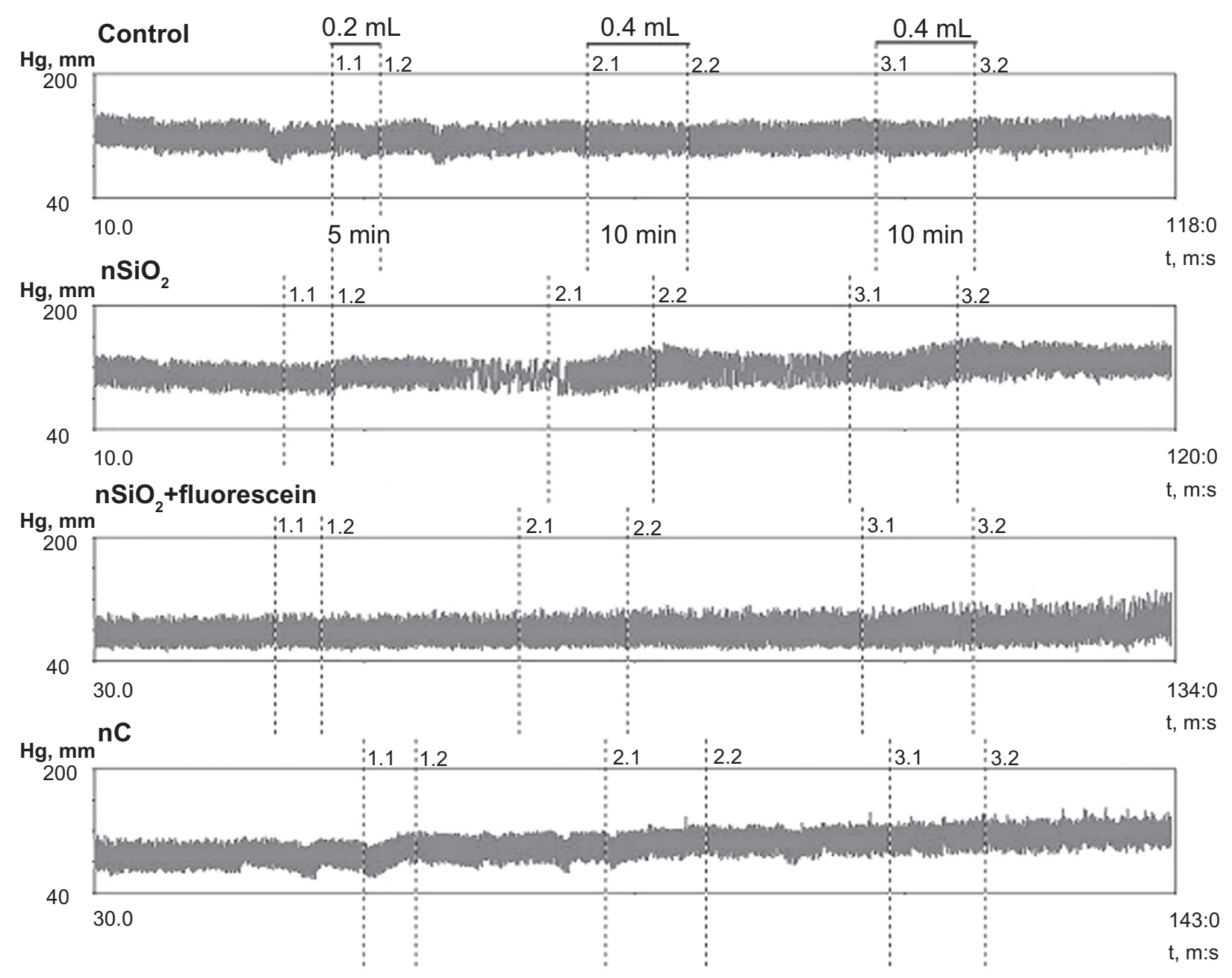

Figure 2 The effect of different nanoparticle formulations on arterial pressure. Abbreviations: $\mathrm{nC}$, carbon nanoparticles; $\mathrm{nSiO}_{2}$, silica.

in the cell culture experiments that mesoporous SNP did not affect cell viability as well as plasma membrane integrity. ${ }^{6}$ Furthermore, mesoporous SNP were recently used for glucoseresponsive controlled release of both insulin and cyclic adenosine monophosphate, ${ }^{7}$ for intracellular delivery of cysteine, ${ }^{8}$ hydrophobic fluorophores, ${ }^{9}$ and genetic constructs. ${ }^{10}$ It should be noted however, that long-term biocompatibility of SNP awaits further investigation in the relevant models. As to the biodegradability of silica nanomaterials, the first evidence on this issue is also encouraging. One recent study suggested that

Table 2 Distribution of fluorescein (FLU)- and indocyanine green (ICG)-labeled silica nanoparticles in different rat organs 30 minutes after nanoparticle administration

\begin{tabular}{|c|c|c|c|c|c|c|}
\hline \multirow[t]{2}{*}{ Organ } & \multicolumn{3}{|c|}{$\lambda=780 \mathrm{~nm}$} & \multicolumn{3}{|l|}{$\lambda=435 \mathrm{~nm}$} \\
\hline & $\begin{array}{l}\text { Control, } \\
\mathrm{FI}^{\mathrm{a}}\end{array}$ & $\begin{array}{l}\text { ICG-labeled } \\
\text { nanoparticles, F2 }\end{array}$ & $\Delta \mathbf{F} \%$ & $\begin{array}{l}\text { Control, } \\
\text { GI }\end{array}$ & $\begin{array}{l}\text { FLU-labeled } \\
\text { nanoparticles, G2 }\end{array}$ & $\Delta \mathbf{G} \%$ \\
\hline Heart & $12,5 \pm 0,5$ & $22,5 \pm 0,5$ & $80,8 \pm 3,9$ & $90,2 \pm 13,2$ & $106,0 \pm 7,0$ & $18,2 \pm 9,3$ \\
\hline Brain & $26,5 \pm 0,5$ & $35,5 \pm 0,5$ & $77, I \pm 7,0$ & $115,0 \pm 7,1$ & $119,5 \pm 13,4$ & $5,5 \pm 2,8$ \\
\hline Liver & $14,5 \pm 0,5$ & $1213,5 \pm 193,0$ & $83 \mid I, 4 \pm 1232,0$ & $94,7 \pm 31,5$ & $113,5 \pm 24,8$ & $22,3 \pm 14,6$ \\
\hline Spleen & $16,0 \pm 9,9$ & $20,0 \pm 4,2$ & $62,1 \pm 27,0$ & $80,7 \pm 10,2$ & $99,0 \pm 7,1$ & $23, I \pm 6,8$ \\
\hline Lung & $29,0 \pm 5,7$ & $127,5 \pm 24,8$ & $356,6 \pm 123,0$ & $87,6 \pm 6,0$ & $\mid 13,5 \pm 2,1$ & $30,0 \pm 1 \mathrm{I}, 4$ \\
\hline Kidney & $16,0 \pm 5,7$ & $164,0 \pm 21,2$ & $1018,3 \pm 373,0$ & $76,3 \pm 0,4$ & $82,2 \pm 6,2$ & $9,3 \pm 6,7$ \\
\hline
\end{tabular}

Notes: ${ }^{\mathrm{a}}$ Total fluorescence intensity. ${ }^{\mathrm{b}}$ Fluorescence intensity restricted to channel G.

Abbreviations: $\Delta \mathrm{F}$, change in fluorescence as a percentage; $\Delta \mathrm{G}$, change in green as a percentage. 
porous sol-gel silica microparticles can be rapidly eliminated from the organism. ${ }^{11}$ The metabolic pathway for silica biodegradation includes erosion of the nanoparticle surface with formation of plasma-soluble silicic acid and its subsequent excretion by the kidney.

In conclusion, targeted delivery of the nanoparticle-bound drugs to the ischemic cardiac muscle seems to be a challenging strategy to treatment of coronary artery disease. Our results suggest that SNP are not toxic during acute intravenous administration. Functionalization of SNP can be reliably achieved by either immobilization or chemical assembly. Infrared fluorophores such as indocyanine green are preferable for fluorescent labeling of SNP and investigation of their biodistribution.

\section{Disclosures}

The authors report no conflicts of interest that are relevant to this study.

\section{References}

1. Ye X, Yang D. Recent advances in biological strategies for targeted drug delivery. Cardiovasc Hematol Disord Drug Targets. 2009;9(3): 206-221.

2. Erhlich P. Collected studies on immunity. New York, NY: John Wiley; 1906; 442-447.

3. Vance JE, Steenbergen R. Metabolism and functions of phosphatidylserine. Prog Lipid Res. 2005;44(4):207-234.
4. Takahama H, Minamino T, Asanuma H, et al. Prolonged targeting of ischemic/reperfused myocardium by liposomal adenosine augments cardioprotection in rats. $J$ Am Coll Cardiol. 2009;53(8):709-717.

5. Matsumura Y, Maeda H. A new concept for macromolecular therapeutics in cancer chemotherapy: mechanism of tumoritropic accumulation of proteins and the antitumor agent SMANCS. Cancer Res. 1986;46:6387-6392.

6. Slowing II, Vivero-Escoto JL, Wu CW, Lin VS. Mesoporous silica nanoparticles as controlled release drug delivery and gene transfection carriers. Adv Drug Deliv Rev. 2008;60(11):1278-1288.

7. Zhao Y, Trewyn BG, Slowing II, Lin VS. Mesoporous silica nanoparticle-based double drug delivery system for glucose-responsive controlled release of insulin and cyclic AMP. J Am Chem Soc. 2009;131(24):8398-8400.

8. Mortera R, Vivero-Escoto J, Slowing II, Garrone E, Onida B, Lin VS. Cell-induced intracellular controlled release of membrane impermeable cysteine from a mesoporous silica nanoparticle-based drug delivery system. Chem Commun (Camb). 2009; (22):3219-3221.

9. Rosenholm JM, Peuhu E, Eriksson JE, Sahlgren C, Linden M. Targeted intracellular delivery of hydrophobic agents using mesoporous hybrid silica nanoparticles as carrier systems. Nano Lett. 2009;9(9): 3308-3311.

10. Xia T, Kovochich M, Liong M, et al. Polyethyleneimine coating enhances the cellular uptake of mesoporous silica nanoparticles and allows safe delivery of siRNA and DNA constructs. ACS Nano. 2009;3(10):3273-3286.

11. Finnie KS, Waller DJ, Perret FL, et al. Biodegradability of sol-gel silica microparticles for drug delivery. J Sol-Gel Sci Technol. 2009;49: $12-18$.
International Journal of Nanomedicine

\section{Publish your work in this journal}

The International Journal of Nanomedicine is an international, peerreviewed journal focusing on the application of nanotechnology in diagnostics, therapeutics, and drug delivery systems throughout the biomedical field. This journal is indexed on PubMed Central, MedLine, CAS, SciSearch ${ }^{\circledR}$, Current Contents ${ }^{\circledR} /$ Clinical Medicine,

\section{Dovepress}

Journal Citation Reports/Science Edition, EMBase, Scopus and the Elsevier Bibliographic databases. The manuscript management system is completely online and includes a very quick and fair peer-review system, which is all easy to use. Visit http://www.dovepress.com/ testimonials.php to read real quotes from published authors. 